\title{
Detection of a Large Arc of Ionized Hydrogen Far Above the Cas OB6 Association: A Superbubble Blowout into the Galactic Halo?
}

\author{
R. J. Reynolds \\ N. C. Sterling \\ L. M. Haffner \\ Department of Astronomy, University of Wisconsin-Madison, 475 North Charter Street, \\ Madison, WI 53706 \\ Electronic Mail: reynolds@astro.wisc.edu, sterling@uwalumni.com, \\ haffner@astro.wisc.edu
}

\begin{abstract}
The Wisconsin H $\alpha$ Mapper (WHAM) Northern Sky Survey has revealed a loop of H II reaching 1300 pc from the Galactic midplane above the Cas OB6 association in the Perseus sprial arm. This enormous feature surrounds and extends far above the "W4 Chimney" identified by Normandeau et al. and appears to be associated with the star formation activity near the W3/W4/W5 H II region complex. The existence of this ionized structure suggests that past episodes of massive star formation have cleared the H I from an enormous volume above the Perseus arm, allowing Lyman continuum photons from O stars near the Galactic midplane to reach into the halo.
\end{abstract}

Subject headings: galaxies: ISM — Galaxy: halo — ISM:bubbles — ISM: general — ISM:HII regions — ISM:structure

\section{Introduction}

The existence of the widespread, warm $\left(10^{4} \mathrm{~K}\right)$ ionized phase of the interstellar medium is thought to be linked closely to star formation activity (e.g., Zurita, Rosas, and Beckman 2000; Mathis 2000). In particular, it appears that hot massive stars, confined primarily to widely separated stellar associations near the Galactic midplane, are somehow able to photoionize a significant fraction of the gas not only in the disk but also within the halo, 1-2 kpc above the midplane; no other source with sufficient ionizing power has been identified (Reynolds 1993). The nature of this disk-halo connection is not understood. For example, 
the need to have a large fraction of the Lyman continuum photons from $\mathrm{O}$ stars travel hundreds of parsecs through the disk seems to conflict with the traditional picture of $\mathrm{H} \mathrm{I}$ permeating much of the interstellar volume near the Galactic plane.

It has been suggested that "superbubbles" of hot gas, especially superbubbles that blow out of the disk ("galactic chimneys"), may sweep large regions of the disk clear of H I, allowing ionizing photons from the $\mathrm{O}$ stars within them to travel unimpeded across these cavities and into the halo (e.g., Norman 1991; Norman and Ikeuchi 1989). Another possibility is that the Lyman continuum radiation itself is able to carve out extensive regions of $\mathrm{H}$ II through low density portions of the H I (e.g., Miller and Cox 1993), perhaps creating photoionized pathways or "warm H II chimneys" that extend far above the midplane (Dove and Shull 1994; Dove, Shull, and Ferrara 2000). Although the existence of superbubbles has long been established (e.g., Heiles 1984), direct observational evidence that such cavities are actually responsible for the transport of hot gas and ionizing radiation up into the Galactic halo is very limited.

The Wisconsin H $\alpha$ Mapper (WHAM) Northern Sky Survey has provided strong evidence for superbubble blowout and for a disk-halo connection that is allowing the transport of Lyman continuum photons from the midplane into the Galactic halo above the "W4 Chimney" in the Perseus spiral arm. These results are presented below.

\section{Observations and Results}

WHAM is a high-throughput Fabry-Perot observing facility dedicated to the detection and study of faint optical emission lines from the diffuse ionized gas in the disk and halo of the Milky Way (Haffner 1999; Tufte 1997; Reynolds, Haffner, and Tufte 1999). It provides a $1^{\circ}$ diameter beam on the sky and produces a $12 \mathrm{~km} \mathrm{~s}^{-1}$ resolution spectrum over a $200 \mathrm{~km} \mathrm{~s}^{-1}$ wide spectral window. This facility is located at Kitt Peak, Arizona, and operated remotely from Madison, Wisconsin. Between 1997 January and 1998 September, WHAM carried out one of its primary missions, a northern sky $\mathrm{H} \alpha$ survey, consisting of 37,300 spectra on a $0.85 \times 0.98$ grid above $-30^{\circ}$ declination. Each spectrum was centered near the LSR velocity and had a sensitivity limit of approximately $0.1 \mathrm{R}$, where $1 \mathrm{R}$ (Rayleigh) is $10^{6} / 4 \pi$ photons $\mathrm{cm}^{-2} \mathrm{~s}^{-1} \mathrm{sr}^{-1}$ or $2.41 \times 10^{-7} \mathrm{erg}^{-2} \mathrm{~cm}^{-1} \mathrm{sr}^{-1}$ at $\mathrm{H} \alpha$. This $\mathrm{H} \alpha$ survey provides for the first time a radial velocity resolved map of the diffuse ionized hydrogen over the sky comparable to earlier $21 \mathrm{~cm}$ surveys of the neutral hydrogen.

Using the Dominion Radio Astrophysical Observatory (DRAO) high angular resolution $21 \mathrm{~cm}$ observations, Normandeau, Taylor, and Dewdney $(1996,1997)$ found an H I cavity 
surrounding the W4 H II region and its associated star cluster ( $\mathrm{OCl} 352)$ in the Cas OB6 association, $2.2 \mathrm{kpc}$ distant in the Perseus arm. Based on its upwardly directed conical appearance in the H I channel maps, Normandeau et al proposed that this cavity is a Galactic chimney extending at least $4^{\circ}(150 \mathrm{pc})$ above the midplane, the limit of the DRAO survey. An $\mathrm{H} \alpha$ image of the region obtained by Dennison, Topasna, and Simonetti (1997) revealed an H II shell associated with the photoionized inner wall of this cavity, which they were able to follow to $7^{\circ}(260 \mathrm{pc})$ from the midplane. The new WHAM survey provides the opportunity to examine the distribution and kinematics of the H II in this region to much higher Galactic latitudes. These observations suggest that the W4 Chimney is the lower end of an enormous cavity that extends at least $30^{\circ}(1300 \mathrm{pc})$ from the midplane.

A portion of the WHAM Northern Sky Survey from Galactic longitude $120^{\circ}$ to $155^{\circ}$ and from the Galactic equator to $+40^{\circ}$ latitude is shown in Figure 1. The lower portion of this $\mathrm{H} \alpha$ map includes the regions of active star formation associated with the W3/W4/W5 H II regions in the Perseus spiral arm (e.g., Carpenter, Heyer, and Snell 2000). The radial velocity interval $-65 \mathrm{~km} \mathrm{~s}^{-1}$ to $-55 \mathrm{~km} \mathrm{~s}^{-1}$ was chosen so that the emission associated with the 2-3 kpc-distant Perseus arm is clearly revealed in the figure, while the emission from the often brighter foreground H II, having radial velocities nearer the LSR, is excluded. A striking feature in Figure 1 is the large $\mathrm{H} \alpha$ loop, arching away from the Galactic plane at $l=132^{\circ}$ and $l=145^{\circ}$ and reaching a maximum latitude of $+30^{\circ}$ near $l=140^{\circ}$. The low angular resolution of the survey $\left(1^{\circ}\right)$ and the limited dynamic range in this display wash out the $\mathrm{H} \alpha$ emission associated with the "W4 Chimney". The small horseshoe shaped feature centered near $l=134^{\circ}, \mathrm{b}=+3^{\circ}$ outlines the location and extent of the $\mathrm{H} \alpha$ emission associated with the Chimney as observed by Dennison et al (1997). The $\mathrm{H} \alpha$ emission line profiles in this region of the sky generally consist of two radial velocity components, one centered near the LSR, associated with relatively local gas within a few hundred parsecs of the sun, and the other centered near -50 to $-60 \mathrm{~km} \mathrm{~s}^{-1}$, associated with the gas in the Perseus arm. This is illustrated in Figure 2, which shows $\mathrm{H} \alpha$ spectra from the WHAM survey in a sequence of six directions cutting across the large loop. In this sequence the $\mathrm{H} \alpha$ emission associated with the loop can be clearly identified as the radial velocity component near $-60 \mathrm{~km} \mathrm{~s}^{-1}$, which is most prominant in the three spectra from $l=133^{\circ} .1, b=+24.6$ to $l=134.5, b=+22^{\circ} .9$, indicating that the loop is approximately $3^{\circ}(100 \mathrm{pc})$ thick at that location.

Tables 1 and 2 list the centroid velocity, width, and intensity of the $\mathrm{H} \alpha$ emission associated with the W4 Chimney and the large $\mathrm{H} \alpha$ loop, respectively. The values are obtained from Gaussian fits to the "Perseus arm component" in the WHAM survey spectra at representative positions along the $\mathrm{H} \alpha$ ridges of the respective loops. Each of the selected directions along the large $\mathrm{H} \alpha$ loop is indicated by an " $\times$ " in Figure 1. The emission measure for each 
of these directions was calculated from the relation

$$
\mathrm{EM}=2.75 \mathrm{I}_{\alpha} \mathrm{T}_{4}^{0.9} \mathrm{e}^{\tau_{\alpha}} \mathrm{cm}^{-6} \mathrm{pc}
$$

where $\mathrm{I}_{\alpha}$ is the $\mathrm{H} \alpha$ intensity in Rayleighs, $\mathrm{T}_{4}$ is the temperature in units of $10^{4} \mathrm{~K}$, and $\tau_{\alpha}$ is the optical depth at $\mathrm{H} \alpha$ due to interstellar extinction. Because this loop is located far from the midplane, behind most of the $\mathrm{H} \mathrm{I}$, we estimated the extinction from the neutral hydrogen column density N(H I) in each direction (Hartmann and Burton 1997), using the relationship between N(H I) and $\tau_{\alpha}$ given by Bohlin et al (1978) and Mathis (1990). The values for $\tau_{\alpha}$ are approximately 0.3 at $b \gtrsim 20^{\circ}$ and rise to about 0.8 for the lower latitude directions. The resulting emission measures are listed in the last column of Table 2 for $\mathrm{T}_{4}=0.8$ (Haffner, Reynolds, and Tufte 1999).

At a distance of $2.2 \mathrm{kpc}$ (see below), the top of the loop extends nearly $1300 \mathrm{pc}$ above the midplane, and the distance between its "feet" (at $11^{\circ}$ latitude) is $500 \mathrm{pc}$. The derived density and mass of this structure depend somewhat on its geometry, whether it is a loop or a shell viewed in projection. The $\mathrm{H} \alpha$ spectra provide little evidence for a complete shell. The weak emission near $-60 \mathrm{~km} \mathrm{~s}^{-1}$ interior to the loop is not brighter than that seen outside the loop, and there is no sign of line splitting characteristic of an expanding shell. Therefore, assuming a loop of cylindrical shape, with an extent along the line of sight equal to its extent on the sky, we find that the rms density of the $\mathrm{H}^{+}$within the loop varies from $0.1 \mathrm{~cm}^{-3}$ in the high latitude, diffuse portion near $l=140^{\circ}, b=+25^{\circ}$ to about $0.2 \mathrm{~cm}^{-3}$ in the brighter lower latitude portion, near $l \approx 132^{\circ}-134^{\circ}$. This implies that the recombination time ranges from about 0.5 to 1.0 million years, the loop's total mass is approximately $1 \times 10^{5} \mathrm{M}_{\odot}$ (above $10^{\circ}$ latitude), and its gravitational potential energy is approximately $3 \times 10^{51} \mathrm{ergs}$.

\section{Discussion}

A close relationship between this $\mathrm{H} \alpha$ loop and the W4 chimney is suggested both by appearance and kinematics. Unfortunately, at Galactic latitudes below about $10^{\circ}$, the surface brightness of the large loop is less than that of the more diffuse ionized gas in the Perseus arm and can no longer be identified as a distinct feature. However, the downwardly cupped geometry of the large loop is strongly suggestive of it being the high latitude complement to the upwardly cupped W4 Chimney. Also, the radial velocities of the two features appear to blend smoothly together. The observed velocities of the large loop range from $-63 \mathrm{~km} \mathrm{~s}^{-1}$ to

$-45 \mathrm{~km} \mathrm{~s}^{-1}$ (Table 2), while in the W4 Chimney the $\mathrm{H} \alpha$ velocities range from $-60 \mathrm{~km} \mathrm{~s}^{-1}$ to $-39 \mathrm{~km} \mathrm{~s}^{-1}$ (Table 1). The uppermost portions of the W4 Chimney $\left(b=+5^{\circ}\right.$ to $\left.+7^{\circ}\right)$ have a mean radial velocity $\left(\approx-50 \mathrm{~km} \mathrm{~s}^{-1}\right)$ that matches that of the lower portions $\left(b<+15^{\circ}\right)$ 
of the large loop. If this interpretation is correct, then the large extent in longitude of the $\mathrm{H} \alpha$ loop relative to that of the W4 Chimney could be a result of "blowout" into the lower density halo beginning at about $7^{\circ}-10^{\circ}(300-400 \mathrm{pc})$ above the midplane (see, for example, Tenorio-Tagle, Różyczka, and Bodenheimer 1990). It is also possible that the broader extent of the $\mathrm{H} \alpha$ loop is the result of it being created by a more distributed source of energy, that is, not just the W4 region, but the entire W3/W4/W5 complex, which extends nearly 200 pc from $l=134^{\circ}$ to $l=139^{\circ}$ at $b \approx+1^{\circ}$.

For the W4 Chimney, the associated H II appears to be a photoionized wall lining the inside surface of the H I cavity (Dennison et al 1997). For the large $\mathrm{H} \alpha$ loop, there is no clearly associated outer $\mathrm{H} \mathrm{I}$ wall. However, along the low longitude side $\left(l=132^{\circ}-134^{\circ}, b=+14^{\circ}\right.$ to $+20^{\circ}$ ), where the $\mathrm{H} \alpha$ velocity is about $-62 \mathrm{~km} \mathrm{~s}^{-1}$, there is a corresponding $\mathrm{H} \mathrm{I}$ "filament" offset approximate $2^{\circ}$ to lower longitude (i.e., to the outside of the loop) on the -62.5 to -55 $\mathrm{km} \mathrm{s}^{-1}$ velocity interval maps of the Dwingeloo $21 \mathrm{~cm}$ survey (Hartmann 1994). This suggests that, as in the W4 Chimney, the H II loop is photoionized by sources within it. Because of the relatively short recombination time $\left(\lesssim 10^{6} \mathrm{yrs}\right)$, the $\mathrm{H} \alpha$ intensity of the loop is then a measure of the current Lyman continuum flux (e.g., Tufte, Reynolds, and Haffner 1998). Ignoring any geometric projection effects, we estimate that the one-sided flux of ionizing radiation incident on the loop ranges from $2.4 \times 10^{6}$ photons $\mathrm{cm}^{-2} \mathrm{~s}^{-1}$ on the brighter, low longitude side, where the H II appears to be ionization bounded, to $0.8 \times 10^{6}$ photons $\mathrm{cm}^{-2}$ $\mathrm{s}^{-1}$ at the top near $l=140^{\circ}, b=+25^{\circ}$. Since there is no detected H I counterpart in this fainter portion, the loop may be density bounded, and therefore the derived value for the incident ionizing flux for that portion must be considered a lower limit. The $\mathrm{H} \alpha$ luminosity of the entire loop implies a total hydrogen ionization rate of $3 \times 10^{48} \mathrm{~s}^{-1}$.

The origin of most of the ionizing radiation appears to be the $\mathrm{OCl} 352$ star cluster $\left(l=134^{\circ} 8, b=+1^{\circ} 0\right)$, which contains the largest concentration of O stars (9) within the W3/W4/W5 complex and also appears to be the most porous to its stars' ionizing radiation (Normandeau et al 1997). The distance to OCl 352 is approximately $2.2 \mathrm{kpc}$ (see Gray et al 1999). The ionization rate of the loop thus requires $1.3 \%$ of the cluster's Lyman continuum luminosity of $2.3 \times 10^{50}$ photons s$^{-1}$ (Basu et al 1999; Dennison et al 1997). However, since the loop subtends only about $0.4 \mathrm{sr}$ as seen by the cluster, this would require that approximately $40 \%$ of this luminosity escape the cluster, if it were escaping isotropically. This is larger than the $15 \%$ estimated by Basu et al (1999), but consistent with the value reported by Terebey et al (2000). It is interesting to note that the Lyman continuum flux required to ionize the loop (i.e., $2.4 \times 10^{6}$ photons $\mathrm{cm}^{-2} \mathrm{~s}^{-1}$ ) would be totally absorbed by an $\mathrm{H}$ I cloud of column density $8 \times 10^{18} / \mathrm{n}_{\mathrm{H}} \mathrm{cm}^{-2}$, where $\mathrm{n}_{\mathrm{H}}$ is the density of the cloud. Thus the presence of this large ionized loop, $1300 \mathrm{pc}$ from the midplane, indicates the existence of an enormous region of the Galactic disk that is remarkably free of H I. Since most of the 
loop appears to be density bounded, the extent of this H I-free region could be even greater than that outlined by the $\mathrm{H} \alpha$.

Dennison et al (1997) have argued that the W4 Chimney is significantly older (age of 6-10 Myr) than the $\mathrm{OCl} 352$ cluster (2 Myr), suggesting that the W4 Chimney was formed by an earlier generation of stars. There has in fact been speculation that this region once contained a Giant Molecular Cloud that was disrupted by an earlier generation of OB stars (Carpenter et al 2000). The H $\alpha$ loop in Figure 1 extends 1000 pc beyond the W4 Chimney and thus must be even older. Numerical simulations of superbubble growth suggest that blowouts into the halo, reaching heights of 1000 pc, may take 10-20 Myr or more (e.g., Tomisaka 1998; Mac Low, McCray, and Norman 1989). Therefore, this entire structure, the loop revealed by WHAM as well as the smaller W4 Chimney below it, seem to be the product not of a single event, but a sequence of star formation episodes in the W3/W4/W5 region over a period of at least 10-20 million years. The latest episode, centered near W4, is reenergizing and ionizing the older, preexisting cavity, making it fluoresce in $\mathrm{H} \alpha$.

\section{Summary and Concluding Remarks}

The large H $\alpha$ loop above the Cas OB6 association in the Perseus arm reveals the existence of a huge $\mathrm{H}$ I-free cavity apparently created by energetic events associated with past star formation activity near the W4 Chimney discovered by Normandeau et al (1996). The cluster $\mathrm{OCl} 352$, containing nine $\mathrm{O}$ stars, has reionized the outer walls of this cavity, revealing its enormous extent on the WHAM H $\alpha$ survey. The morphology of this entire structure appears similar to that predicted by numerical simulations of expanding superbubbles that have reached "blowout" into the galactic halo.

If such H I-free cavities are common, they could explain how Lyman continuum photons from $\mathrm{O}$ stars near the midplane are able to ionize large regions of the disk and halo. However, these cavities would be difficult to identify, since their $\mathrm{H} \alpha$ intensity (the evidence that they are in fact a conduit for ionizing radiation) depends upon the Lyman continuum luminosity of the stars that are currently within them. A cavity 1000 pc or more in size containing one or two $\mathrm{O}$ stars would have an $\mathrm{H} \alpha$ surface brightnesses of only $\sim 0.1 \mathrm{R}$, which would be lost in the brighter, more diffuse $\mathrm{H} \alpha$ background. Nevertheless, the presence of other large ionized loops revealed by the WHAM survey (e.g., Haffner, Reynolds, and Tufte 1998) does suggest that this cavity above the Perseus arm is not unique.

This work, including the operation of WHAM, was supported by the National Science Foundation through grant AST96 19424. 


\section{REFERENCES}

Basu, S., Johnstone, D., \& Martin, P. G. 1999, ApJ, 516, 843

Bohlin, R. C., Savage, B. D., \& Drake, J. F. 1978, ApJ, 224, 132

Carpenter, J. M., Heyer, M. H., \& Snell, R. L. 2000, ApJS, 130, 381

Dennison, B., Topasna, G. A., \& Simonetti, J. H. 1997, ApJ, 474, L31

Dove, J. B., \& Shull, J. M. 1994, ApJ, 423, 196

Dove, J. B., Shull, J. M., \& Ferrara, A. 2000, ApJ, 531, 846

Gray, A. D., Landecker, T. L., Dewdney, P. E., Taylor, A. R., Willis, A. G., \& Normandeau, M. 1999, ApJ, 514, 221

Haffner, L. M. 1999, PhD Thesis, University of Wisconsin-Madison

Haffner, L. M., Reynolds, R. J., \& Tufte, S. L. 1998, ApJ, 501, L83

Haffner, L. M., Reynolds, R. J., \& Tufte, S. L. 1999, ApJ, 523, 223

Hartmann, D. 1994, Doctoral Thesis, Leiden University

Hartman, D., \& Burton, W. B. 1997, Atlas of Galactic Neutral Hydrogen, (Cambridge; New York: Cambridge University Press)

Heiles, C. 1984, ApJS, 55, 585

Mac Low, M.-M., McCray, R., \& Norman, M. 1989, ApJ, 337, 141

Mathis, J. S. 1990, ARA\&A, 28, 37

Mathis, J. S. 2000, ApJ, 544, 347

Miller, W. W., III, \& Cox, D. P. 1993, ApJ, 417, 579

Norman, C. A. 1991, in The Interstellar Disk-Halo Connection in Galaxies, IAU Symp. No. 144, ed. H. Bloemen, (Dordrect:Kluwer), p. 337

Norman, C. A., \& Ikeuchi, S. 1989, ApJ, 345, 372

Normandeau, M., Taylor, A. R., \& Dewdney, P. E. 1996, Nature, 380, 687

Normandeau, M., Taylor, A. R., \& Dewdney, P. E. 1997, ApJS, 108, 279

Reynolds, R. J. 1993, in Massive Stars: Their Lives in the Interstellar Medium, ASP Conf. Ser., Vol. 35, eds., J. P. Cassinelli and E. B. Churchwell (San Francisco:APS), p. 338

Reynolds, R. J., Haffner, L. M., \& Tufte, S. L. 1999, in New Perspectives on the Interstellar Medium, ASP Conf. Ser., Vol. 168, eds., A. R. Taylor, T. L. Landecker, \& G. Joncas (San Francisco:ASP), p. 149 
Tenorio-Tagle, G., Różyczka, M., \& Bodenheimer, P. 1990, A\&A, 237, 207

Terebey, S., Fich, M., Taylor, R., Cao, Y., \& Hancock, T. 2000, BAAS, 196, 26.09

Tomisaka, K. 1998, MNRAS, 298, 797

Tufte, S. L. 1997, PhD Thesis, University of Wisconsin-Madison

Tufte, S. L., Reynolds, R. J., \& Haffner, L. M. 1998, ApJ, 504, 773

Zurita, A., Rosas, M., \& Beckman, J. E. 2000, A\&A, 363, 9

This preprint was prepared with the AAS IATEX macros v5.0. 
Table 1. H $\alpha$ Parameters for the W4 Superbubble

\begin{tabular}{cccc}
\hline \hline $\begin{array}{c}\text { Direction } \\
(l, b)\end{array}$ & $\begin{array}{c}\mathrm{V}_{\mathrm{LSR}} \\
\left(\mathrm{km} \mathrm{s}^{-1}\right)\end{array}$ & $\begin{array}{c}\text { FWHM } \\
\left(\mathrm{km} \mathrm{s}^{-1}\right)\end{array}$ & $\begin{array}{c}\text { Intensity } \\
(\mathrm{R})^{\mathrm{a}}\end{array}$ \\
\hline $134.8,+0.9$ & $-45 \pm 1$ & $29 \pm 3$ & $277 \pm 19$ \\
$135.3,+1.7$ & $-43 \pm 1$ & $29 \pm 3$ & $142 \pm 10$ \\
$135.9,+2.6$ & $-41 \pm 1$ & $30 \pm 3$ & $43.3 \pm 3.0$ \\
$136.5,+3.4$ & $-42 \pm 1$ & $29 \pm 3$ & $14.2 \pm 1.0$ \\
$136.1,+4.2$ & $-41 \pm 1$ & $30 \pm 3$ & $10.6 \pm 0.8$ \\
$135.8,+5.1$ & $-48 \pm 3$ & $36 \pm 5$ & $4.6 \pm 0.8$ \\
$135.5,+5.9$ & $-59 \pm 2$ & $28 \pm 3$ & $2.4 \pm 0.3$ \\
$133.2,+6.8$ & $-46 \pm 3$ & $38 \pm 4$ & $2.1 \pm 0.4$ \\
$133.5,+5.9$ & $-47 \pm 2$ & $33 \pm 3$ & $3.1 \pm 0.4$ \\
$132.8,+5.1$ & $-46 \pm 1$ & $33 \pm 3$ & $6.9 \pm 0.5$ \\
$133.2,+4.2$ & $-41 \pm 3$ & $49 \pm 5$ & $4.4 \pm 0.8$ \\
$132.5,+3.4$ & $-40 \pm 1$ & $34 \pm 3$ & $4.9 \pm 0.6$ \\
$132.9,+2.6$ & $-39 \pm 1$ & $31 \pm 3$ & $14.0 \pm 1.0$ \\
$134.3,+1.7$ & $-43 \pm 1$ & $28 \pm 3$ & $79.9 \pm 5.6$ \\
\hline
\end{tabular}

${ }^{\mathrm{a}} 1 \mathrm{R}=10^{6} / 4 \pi$ photons $\mathrm{cm}^{-2} \mathrm{~s}^{-1} \mathrm{sr}^{-1}$, or $2.41 \times$ $10^{-7}$ erg $\mathrm{cm}^{-2} \mathrm{~s}^{-1} \mathrm{sr}^{-1}$ at $\mathrm{H} \alpha$. 
Table 2. Parameters for the Large $\mathrm{H} \alpha$ Loop

\begin{tabular}{ccccc}
\hline \hline $\begin{array}{c}\text { Direction } \\
(l, b)\end{array}$ & $\begin{array}{c}\mathrm{V}_{\mathrm{LSR}} \\
\left(\mathrm{km} \mathrm{s}^{-1}\right)\end{array}$ & $\begin{array}{c}\text { FWHM } \\
\left(\mathrm{km} \mathrm{s}^{-1}\right)\end{array}$ & $\begin{array}{c}\text { Intensity } \\
(\mathrm{R})^{\mathrm{a}}\end{array}$ & $\begin{array}{c}\mathrm{EM} \\
\left(\mathrm{cm}^{-6} \mathrm{pc}\right)\end{array}$ \\
\hline $144.3,+11.0$ & $-48 \pm 2$ & $24^{\mathrm{b}}$ & $0.72 \pm 0.09$ & $3.8 \pm 0.5$ \\
$146.1,+19.5$ & $-54 \pm 4$ & $38^{\mathrm{b}}$ & $0.70 \pm 0.12$ & $2.2 \pm 0.4$ \\
$144.9,+22.1$ & $-56 \pm 6$ & $30 \pm 5$ & $0.47 \pm 0.09$ & $1.5 \pm 0.3$ \\
$142.4,+23.8$ & $-53 \pm 5$ & $26^{\mathrm{b}}$ & $0.38 \pm 0.14$ & $1.2 \pm 0.5$ \\
$140.0,+25.5$ & $-58 \pm 5$ & $27 \pm 3$ & $0.38 \pm 0.13$ & $1.1 \pm 0.4$ \\
$137.4,+24.6$ & $-56 \pm 5$ & $37 \pm 7$ & $0.81 \pm 0.17$ & $2.5 \pm 0.6$ \\
$134.9,+23.8$ & $-51 \pm 1$ & $29 \pm 5$ & $1.27 \pm 0.17$ & $3.7 \pm 0.5$ \\
$133.2,+22.1$ & $-42 \pm 1$ & $31 \pm 4$ & $1.76 \pm 0.16$ & $5.3 \pm 0.5$ \\
$133.6,+19.5$ & $-63 \pm 3$ & $23^{\mathrm{b}}$ & $0.74 \pm 0.16$ & $2.4 \pm 0.6$ \\
$133.8,+17.8$ & $-60 \pm 2$ & $25 \pm 3$ & $1.07 \pm 0.16$ & $3.6 \pm 0.6$ \\
$132.0,+14.4$ & $-62 \pm 4$ & $29^{\mathrm{b}}$ & $0.77 \pm 0.17$ & $3.4 \pm 0.8$ \\
$132.2,+11.9$ & $-45 \pm 3$ & $31^{\mathrm{b}}$ & $0.93 \pm 0.17$ & $5.1 \pm 1.0$ \\
\hline
\end{tabular}

${ }^{\mathrm{a}} 1 \mathrm{R}=10^{6} / 4 \pi$ photons $\mathrm{cm}^{-2} \mathrm{~s}^{-1} \mathrm{sr}^{-1}$, or $2.41 \times 10^{-7} \mathrm{erg} \mathrm{cm}^{-2}$ $\mathrm{s}^{-1} \mathrm{sr}^{-1}$ at $\mathrm{H} \alpha$.

${ }^{b}$ Due to blending, the width of this component is highly uncertain. An average width has been fixed in order to determine $\mathrm{V}_{\mathrm{LSR}}$ and the intensity. 


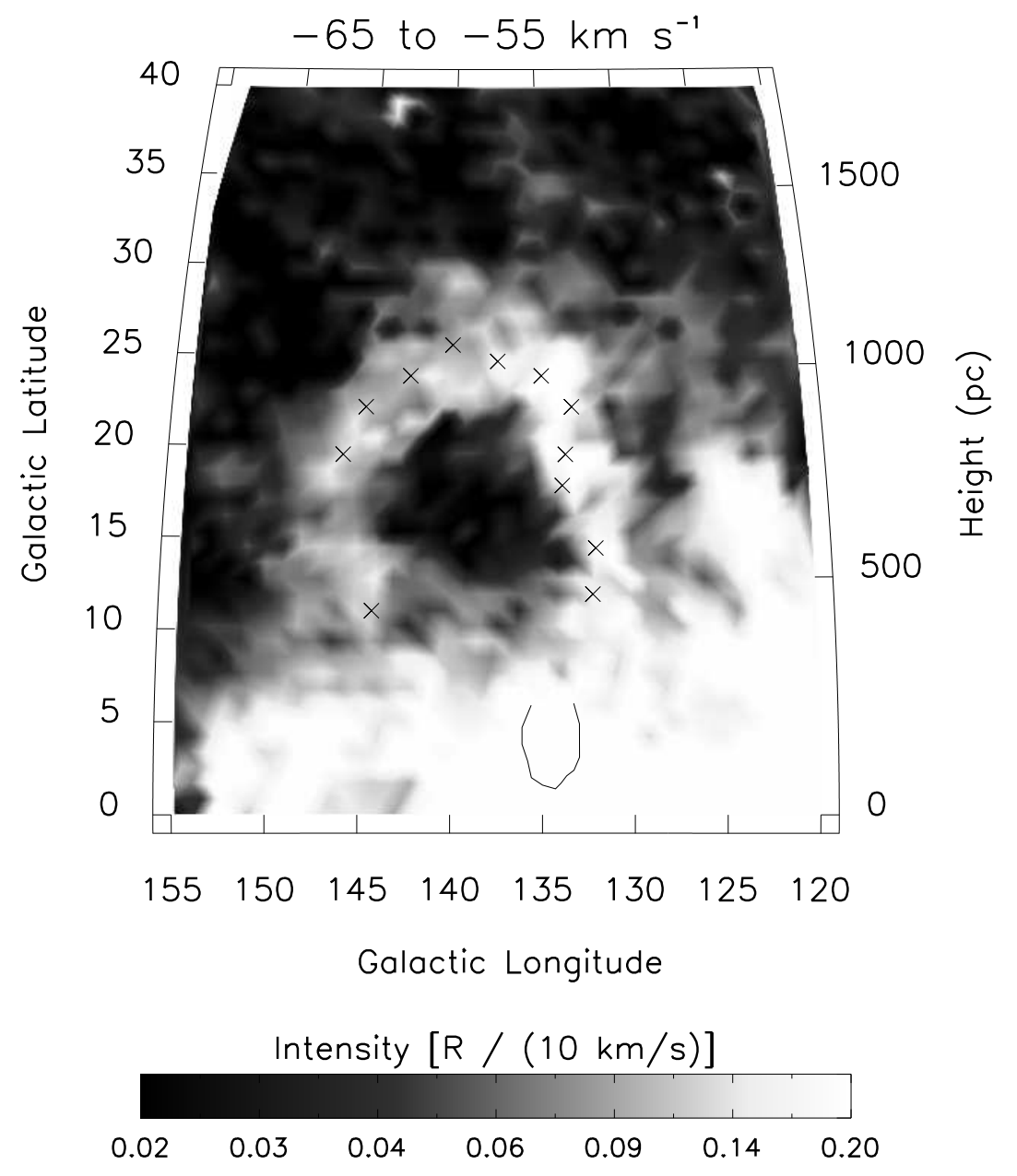

Fig. 1.- A map of ionized interstellar hydrogen above the Perseus spiral arm in the velocity interval $-65 \mathrm{~km} \mathrm{~s}^{-1}$ to $-55 \mathrm{~km} \mathrm{~s}^{-1}$ (from the WHAM Northern Sky Survey). The horseshoeshaped figure near $l=135^{\circ}, b=+3^{\circ}$ indicates the location of the W4 Chimney identified by Normandeau et al (1996) and Dennison et al (1997). Directions listed in Table 2 are denoted by an " $x$ ". The intensity scale is logarithmic. The vertical scale on the right side of the map denotes the height above the midplane for a distance of $2.2 \mathrm{kpc}$. 


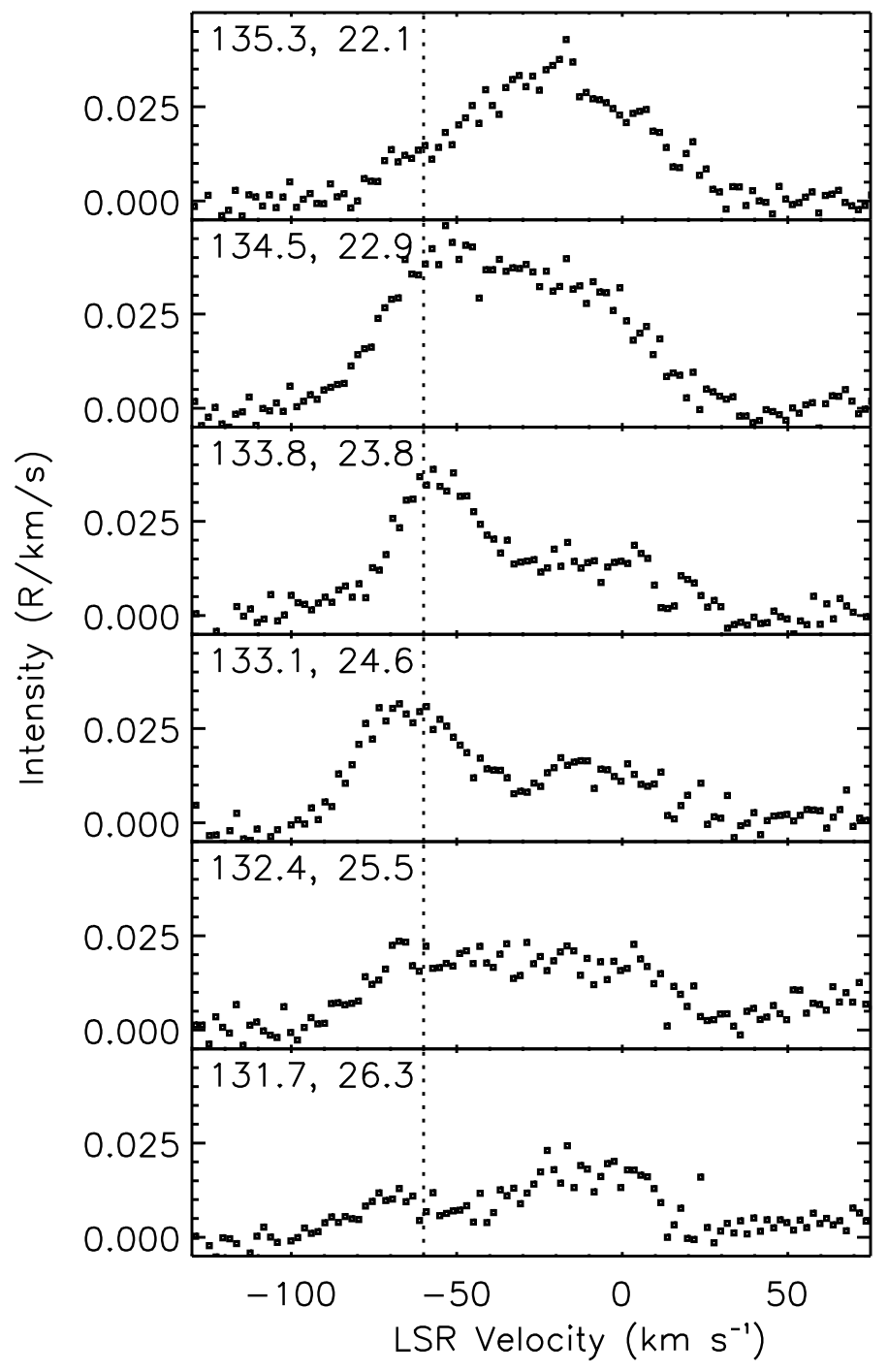

Fig. 2.- Spectra from the WHAM Sky Survey showing the $\mathrm{H} \alpha$ line profiles at six positions that cut across the large $\mathrm{H} \alpha$ loop near $l=133^{\circ}, b=+24^{\circ}$. Emission from the Perseus arm is associated with the velocity component centered near $-60 \mathrm{~km} \mathrm{~s}^{-1}$ (vertical dotted line). 